Research Article

\title{
UGT1A1 Polymorphism for Irinotecan Dose Escalation in Patients with BRAF-Mutated Metastatic Colorectal Cancer Treated with First-Line Bevacizumab and FOLFIRI
}

\author{
Yi-Chien Hsieh $\mathbb{D}^{1,2}$ Tsung-Kun Chang, ${ }^{1,2}$ Wei-Chih Su, ${ }^{1,2}$ Ching-Wen Huang, ${ }^{1,3}$ \\ Hsiang-Lin Tsai, ${ }^{1,3}$ Yen-Cheng Chen, ${ }^{1,2}$ Ching-Chun Li, ${ }^{1,2}$ Po-Jung Chen, ${ }^{1,4}$ \\ Tzu-Chieh Yin, ${ }^{1,5}$ Cheng-Jen Ma, ${ }^{1,6}$ and Jaw-Yuan Wang $\mathbb{1}^{1,2,3,7,8,9}$ \\ ${ }^{1}$ Division of Colorectal Surgery, Department of Surgery, Kaohsiung Medical University Hospital, Kaohsiung Medical University, \\ Kaohsiung, Taiwan \\ ${ }^{2}$ Graduate Institute of Clinical Medicine, College of Medicine, Kaohsiung Medical University, Kaohsiung, Taiwan \\ ${ }^{3}$ Department of Surgery, Faculty of Medicine, College of Medicine, Kaohsiung Medical University, Kaohsiung, Taiwan \\ ${ }^{4}$ Division of Colorectal Surgery, Department of Surgery, Kaohsiung Municipal Hsiaokang Hospital, Kaohsiung, Taiwan \\ ${ }^{5}$ Department of Surgery, Kaohsiung Municipal Tatung Hospital, Kaohsiung Medical University, Kaohsiung, Taiwan \\ ${ }^{6}$ Division of Digestive and General Surgery, Department of Surgery, Kaohsiung Medical University Hospital, \\ Kaohsiung Medical University, Kaohsiung, Taiwan \\ ${ }^{7}$ Graduate Institute of Medicine, College of Medicine, Kaohsiung Medical University, Kaohsiung, Taiwan \\ ${ }^{8}$ Center for Cancer Research, Kaohsiung Medical University, Kaohsiung, Taiwan \\ ${ }^{9}$ Cohort Research Center, Kaohsiung Medical University, Kaohsiung, Taiwan
}

Correspondence should be addressed to Jaw-Yuan Wang; cy614112@ms14.hinet.net

Received 15 November 2020; Revised 12 February 2021; Accepted 25 February 2021; Published 11 March 2021

Academic Editor: Yuan Seng Wu

Copyright (c) 2021 Yi-Chien Hsieh et al. This is an open access article distributed under the Creative Commons Attribution License, which permits unrestricted use, distribution, and reproduction in any medium, provided the original work is properly cited.

Background. Patients with metastatic colorectal cancer (mCRC) and BRAF V600E mutation have a poor prognosis, with a median progression-free survival (PFS) of only 5-7 months after initial therapy. The current standard first-line chemotherapy for these patients includes FOLFOX or FOLFIRI plus bevacizumab. In this study, we explored the effects and oncological outcomes of UGT1A1 polymorphism for irinotecan escalation in patients with BRAF-mutated mCRC. Patients and Methods. This retrospective study included 17 patients with BRAF-mutated mCRC between April 2016 and December 2019. UGT1A1 genotyping was performed on all patients prior to initiating bevacizumab plus FOLFIRI chemotherapy. The primary endpoint was PFS, and the secondary endpoints were toxicity, response rate, disease control rate, and overall survival (OS). Results. Fifteen and two patients had $U G T 1 A 11 * / 1 *$ and $1 * / 28 *$, respectively. Eight underwent irinotecan dose escalation with tolerable adverse effects (AEs), and nine maintained an irinotecan dose of $180 \mathrm{mg} / \mathrm{m}^{2}$ or required deescalation to $150 \mathrm{mg} / \mathrm{m}^{2}$ due to intolerable AEs. After a median follow-up period of 15.7 (range, 3-54) months, the median PFS and OS were 9.4 and 15.7 months, respectively. Grade 3/4 AEs were observed in three (6\%) patients. The disease control and partial response rates were $64.7 \%$ and $11.8 \%$, respectively, indicating that most patients $(14,82.3 \%)$ could maintain this as a first-line line therapy with stable disease or proceed to second-line therapy if disease progression occurred, thereby maintaining acceptable performance status. Conclusions. The oncological outcomes of patients with BRAF-mutated mCRC treated using FOLFIRI plus bevacizumab with irinotecan dose escalation as a first-line therapy are acceptable with tolerable AEs; this may be a feasible treatment option in such patients. Pretherapeutic UGT1A1 genotyping-guided dose adjustment can achieve favorable outcomes. 


\section{Introduction}

Colorectal cancer (CRC) is the third most common malignancy and cause of cancer-related deaths worldwide, with approximately $25 \%$ of patients with CRC presenting with metastatic lesions at initial diagnosis [1-3]. Despite advances in systemic chemotherapy, target therapy, and surgical techniques, the median overall survival (OS) of patients with metastatic CRC (mCRC) is approximately 30 months $[4,5]$. Studies have increasingly focused on the molecular pathology and genetic aberrations involved in CRC to stratify patients for individualized treatments [6]. For instance, patients with CRC who have RAS mutations are excluded from treatments using antiepidermal growth factor receptor (EGFR) monoclonal antibody [7, 8]. Among these new findings, $B R A F$ proto-oncogene mutation, a marker of poor prognosis, was observed in $8 \%-15 \%$ of patients with mCRC exhibiting aggressive tumor biology and poor response to standard therapy $[1,2,6,9,10]$.

The $B R A F$ oncogene encodes a serine/threonine kinase downstream of RAS in the MAPK pathway, playing a key role in the regulation of cellular proliferation [11]. Most $B R A F$ mutations are found in a single amino acid substitution in codon 600 of exon 15 (V600E), and they are associated with unique clinical characteristics, including female sex, older age, right-sided tumor, and peritoneal and distant lymph node metastasis $[6,12,13]$.

The current standard first-line chemotherapy for patients with $B R A F$-mutated mCRC includes fluoropyrimidine-based doublet chemotherapy-FOLFOX or FOLFIRI-plus bevacizumab; this regimen affords a median progression-free survival (PFS) of 5-7 months [14]. The phase 3 TRIBE study revealed a trend toward improved OS in patients with $B R A F$ mutation receiving triplet chemotherapy FOLFOXIRI plus bevacizumab although no statistical significance was observed [15]. Because toxicity management remains a concern, the use of FOLFOXIRI as a first-line treatment option reflects that patients with $\mathrm{mCRC}$ may not exhibit adequate performance status to maintain the treatment. Our previous study revealed that patients with mCRC receiving escalated doses of irinotecan based on uridine diphosphate glucuronosyltransferase 1A1 (UGT1A1) genotyping exhibited favorable clinical responses and outcomes $[3,16,17]$. In the present retrospective study, we used real-world data and explored the effects and oncological outcomes of FORFIRI plus bevacizumab as first-line therapy with irinotecan dose escalation according to UGT1A1 polymorphism in patients with $B R A F$-mutated mCRC.

\section{Material and Methods}

2.1. Patients. This retrospective chart review identified 24 patients with histologically or radiologically proven mCRC with BRAF mutation between April 2016 and December 2019. All patients underwent UGT1A1 genotyping before the bevacizumab plus FOLFIRI regimen was started [3]. The recommended starting dose of irinotecan was $180 \mathrm{mg} / \mathrm{m}^{2}$. The dose was escalated in increments of $20-30 \mathrm{mg} / \mathrm{m}^{2}$ until grade $3 / 4$ AEs occurred, depending on the UGT1A1 genotype. We analyzed the response rate, disease control rate (DCR), PFS, OS, and grade $3 / 4$ AEs.

\section{Methods}

3.1. BRAF Mutation Analysis. We extracted DNA from formalin-fixed, paraffin-embedded (FFPE) CRC tissue samples for clinical BRAF mutation analysis by direct sequencing. The FFPE samples were deparaffinized and airdried; subsequently, DNA was isolated using the proteinase $\mathrm{K}$ and QIAamp DNA Micro Kit (QIAGEN) in accordance with the manufacturer's instructions. We designed a set of primers for high-resolution melting (HRM) analysis that were specific for the BRAF V600E mutation while fulfilling the requirements of the LightCycler ${ }^{\circledR} 480$ System Gene Scanning Assay. Primer3 free software was used to design the primers used in this study. The forward and reverse primer sequences were 5'-CATAATGCTTGCTCTGATAGGAAA-3' and 5' ${ }^{\prime}$-TCAGCACATCTCAGGGCCAAA-3', respectively. All the primers synthesized were of standard molecular biology quality (Protech Technology Enterprise Co., Ltd., Taiwan).

Polymerase chain reaction (PCR) was performed with $10 \mu \mathrm{L}$ as the final volume by using a Light Cycler ${ }^{\circledR} 480$ High-Resolution Melting Master (Reference 04909631001, Roche Diagnostics) with $1 \times$ buffer (containing Taq polymerase, nucleotides, and ResoLight dye) and 20 ng of DNA. The primers and $\mathrm{MgCl}_{2}$ were used at concentrations of $0.25 \mu \mathrm{M}$ and $2.5 \mathrm{mM}$, respectively, to identify BRAF mutation status. The HRM analyses were performed using the LightCycler ${ }^{\circledR} 480$ Instrument (Roche Diagnostics) provided with the software LightCycler ${ }^{\circledR} 480$ Gene Scanning Software (version 1.5; Roche Diagnostics). The PCR program required an SYBR Green I filter $(533 \mathrm{~nm})$. It comprised an initial denaturation-activation step at $95^{\circ} \mathrm{C}$ for $10 \mathrm{~min}$, followed by a 45 -cycle program (denaturation at $95^{\circ} \mathrm{C}$ for $15 \mathrm{~s}$, annealing at 60 or $62^{\circ} \mathrm{C}$ for $15 \mathrm{~s}$, and elongation at $72^{\circ} \mathrm{C}$ for $15 \mathrm{~s}$ with fluorescence reading; acquisition mode: single). The melting program included the following three steps: denaturation at $95^{\circ} \mathrm{C}$ for $1 \mathrm{~min}$, renaturation at $40^{\circ} \mathrm{C}$ for $1 \mathrm{~min}$, and subsequent melting that consisted of continuous fluorescence reading from 60 to $90^{\circ} \mathrm{C}$ at a rate of 25 acquisitions per $1^{\circ} \mathrm{C}$.

After HRM analysis, the samples were purified using a PCR-M ${ }^{\mathrm{TM}}$ clean-up system (Viogen, Sunnyvale, CA, USA). The PCR products generated after HRM were directly sequenced. The sequencing reaction was performed at a final volume of $10 \mu \mathrm{L}$, including $1 \mu \mathrm{L}$ of the purified PCR product, $2.5 \mu \mathrm{M}$ of one of the PCR primers, $2 \mu \mathrm{L}$ of the ABI PRISM terminator cycle sequencing kit v3.1 (Applied Biosystems, Foster City, CA, USA), and $2 \mu \mathrm{L}$ of $5 \times$ sequence buffer. The sequencing program is a 25 -cycle PCR program (denaturation at $96^{\circ} \mathrm{C}$ for $10 \mathrm{~s}$, annealing at $50^{\circ} \mathrm{C}$ for $5 \mathrm{~s}$, and elongation at $60^{\circ} \mathrm{C}$ for $\left.4 \mathrm{~min}\right)$. Sequence detection was performed using the ABI Prism 3130 Genetic Analyzer (Applied Biosystems) according to standard protocols [18].

3.2. UGT1A1 Mutation Analysis. Constitutional gene polymorphisms were analyzed with DNA extraction from $4 \mathrm{~mL}$ of peripheral blood by using the Puregene DNA Isolation Kit 
(Gentra Systems Inc., Minneapolis, MN, USA). The patients' genomic DNA was analyzed using direct sequencing to determine the genotype of the UGT1A1 promoter region. The primers used in this study were designed using Primer3. The forward and reverse primer sequences were $5^{\prime}$-AGTCACGTGACACAGTCAAACA- $3^{\prime}$ and $5^{\prime}$ CTTTGCTCCTGCCAGAGGTT- ${ }^{\prime}$, respectively. The PCR volume was $40 \mu \mathrm{L}$, and the PCR conditions for glutathione S-transferase pi 1 (GSTP1) were as follows: $94.0^{\circ} \mathrm{C}$ for $5 \mathrm{~min}$, 30 cycles of denaturation for $30 \mathrm{~s}$ at $94.0^{\circ} \mathrm{C}$, annealing for $20 \mathrm{~s}$ at $67.5^{\circ} \mathrm{C}$, primer extension for $20 \mathrm{~s}$ at $72.0^{\circ} \mathrm{C}$, and final extension for $10 \mathrm{~min}$ at $72.0^{\circ} \mathrm{C}$. We performed a fragment analysis of the PCR products to verify the genotypes through automated capillary electrophoresis by using the ABI PRISM 310 Genetic Analyzer (Applied Biosystems, Foster City, CA, USA), and the genotypes were analyzed using GeneScan and Genotyper (Applied Biosystems) [19].

3.3. Postchemotherapy Surveillance. The treatment response was assessed using computed tomography, magnetic resonance imaging, or positron emission tomography, and the best responses were recorded. The first response assessment was usually performed after the sixth cycle for patients who received bevacizumab combined with FOLFIRI. The criteria of the Response Evaluation Criteria in Solid Tumors (RECIST; version 1.1) [20] were used to classify patient responses. The AEs were monitored and graded in each cycle according to the National Cancer Institute Common Terminology Criteria for Adverse Events (version 4.3; http:// ctep.cancer.gov/reporting/ctc.html). The most effective response was defined as the best result recorded by the investigators. The median follow-up period was 14.5 (range, 3-54) months. This study was approved by the Institutional Review Board of Kaohsiung Medical University Hospital (KMUHIRB-2012-03-02(II)). All patients signed an informed consent form. PFS was the primary endpoint, and the secondary endpoints were toxicity, response rate, DCR, and OS.

3.4. Statistical Analysis. SPSS (version 19.0; SPSS, Chicago, IL, USA) was used for all data analyses. PFS was defined as the time from the initiation of study treatment to the first radiological progression or tumor-related death, whichever came first. OS was defined as the time from initiation of study treatment to death from any cause. The Kaplan-Meier method was used to calculate PFS and OS, and a log-rank test was used to compare time-to-event distributions. Statistical significance was set to $P<0.05$.

\section{Results}

We retrospectively reviewed 24 patients with $B R A F$-mutated mCRC. Seven were excluded for the following reasons: one exhibited BRAF mutation at position 597 (p.L597P); two showed poor performance status (Eastern Cooperative Oncology Group performance status $(\mathrm{ECOG})>2$ ), with intolerance to systemic therapy; and four received different regimens (Figure 1). The median age of the 17 included patients ( 8 men and 9 women) was 56 (range, 35-81) years. Nine and eight patients presented with right- and left-sided CRC, respectively. Thirteen (76.5\%) of the tumors were moderately differentiated, and four $(23.5 \%)$ were poorly differentiated. The most common metastatic site was the liver (12 of 17, 70.5\%) followed by the peritoneum (7, 41.2\%), lung $(5,29.4 \%)$, and distant lymph nodes $(4,23.5 \%)$. Six (35.3\%) patients had single-site metastasis, and 11 (64.7\%) had multiple metastatic sites. Fifteen (88.2\%) and two (11.8\%) patients had UGT1A1 $1 * / 1 *$, respectively (Table 1 ).

Eight $(47.1 \%)$ patients underwent irinotecan dose escalation with tolerable AEs, and nine $(52.9 \%)$ were maintained at the recommended irinotecan dose $\left(180 \mathrm{mg} / \mathrm{m}^{2}\right)$ or switched to a lower dose $\left(150 \mathrm{mg} / \mathrm{m}^{2}\right)$ because of intolerable AEs. No significant differences were noted between the two groups (Table 2). In total, 5 (29.4\%), 10 (58.8\%), and 2 $(11.8 \%)$ patients experienced grade 1, 2, and 3 toxicities, respectively. No grade $4 / 5$ toxicities were reported. The most frequently reported AEs (any grade) included anemia $(76.5 \%)$, fatigue $(58.8 \%)$, nausea $(52.9 \%)$, leukopenia (52.9\%), and hair loss (47\%). Grade 3 AEs were observed in two (11.8\%) patients (one with grade 3 nausea/vomiting, one with grade 3 leukopenia; Table 3 ).

The DCR was $64.7 \%$ (11 of 17 patients), and the response rate was $11.8 \%$ ( 2 of 17 , Table 1 ). The median PFS and OS were 9.4 and 15.7 months, respectively (Figures 2(a) and 2(b)). Regarding tumor sidedness, the median PFS was 4.2 and 12.6 months in patients with left-sided and right-sided mCRC, respectively $(P=0.08$, HR: $0.384, \quad 95 \%$ CI: $0.127-1.159$, Figure $3(\mathrm{a})$ ), whereas the median OS was 11.6 and 16.5 months in patients with left-sided $\mathrm{mCRC}$ and those with right-sided $\mathrm{mCRC}$, respectively $(P=0.293$, HR: 0.438, 95\% CI: 0.094-2.041, Figure 3(b)). Although the differences were not significant, favorable PFS trends were observed in patients with right-sided mCRC. Regarding irinotecan escalation, the median PFS was 11.5 and 5.7 months in patients with and without dose escalation, respectively $(P=0.552$, 95\% CI: 7.307-21.485, Figure 4(a)), whereas the median OS was 15.8 and 14.5 months in patients with and without escalation, respectively ( $P=0.40,95 \%$ CI: $18.241-32.559$, Figure 4(b)). Although no significant difference in PFS and OS was observed between the two groups, a trend of improved PFS was found in patients in the escalation group.

\section{Discussion}

Patients with $B R A F$-mutated mCRC have aggressive tumor biology and poor prognosis. Several clinical trials have been performed to improve survival and DCR [14, 21]. Here, we retrospectively reviewed the clinical characteristics, treatment regimens of FOLFIRI with irinotecan dose escalation plus bevacizumab, toxicities, and oncological outcomes of patients with $B R A F$-mutated $\mathrm{mCRC}$ at a single tertiary center in a real-world setting.

No significant sex, primary tumor location, or metastatic site differences were observed in our study, which was inconsistent with the results of a previous study [13]. Although some studies have reported that older age, female sex, rightsided tumor, and peritoneal metastasis are associated with 


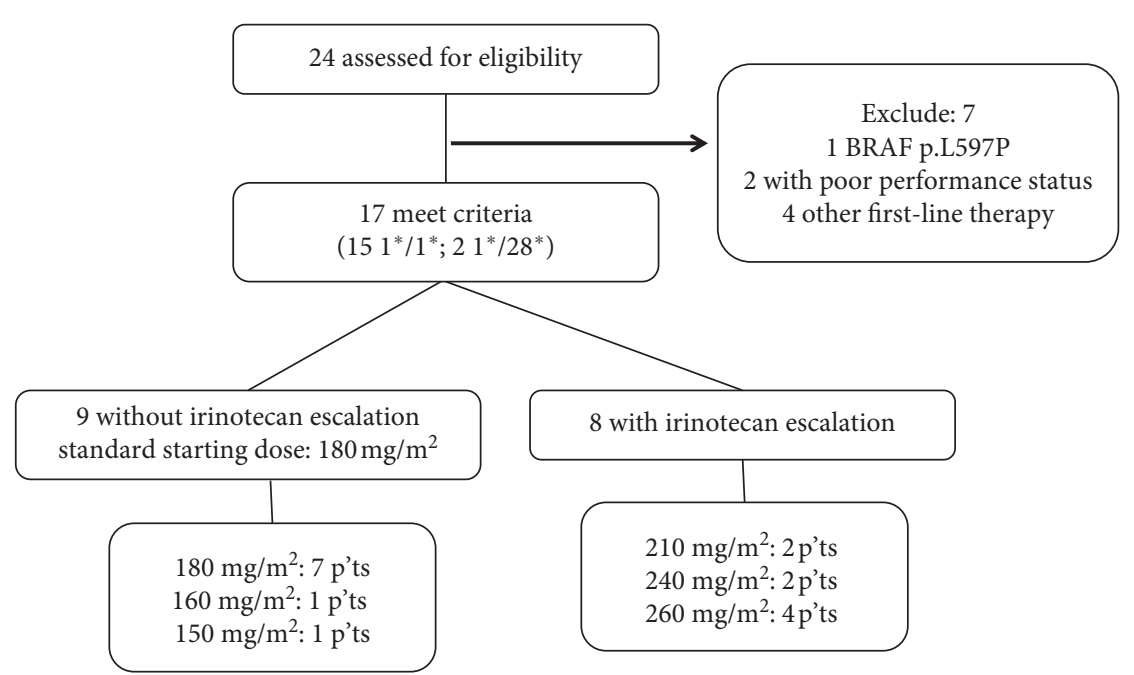

FIGURE 1: Flowchart of patient selection.

TABLE 1: Baseline characteristics of 17 patients with BRAF-mutated mCRC stratified by tumor sidedness.

\begin{tabular}{|c|c|c|c|c|}
\hline Characteristic & All patients $(N=17)$ & Group A (right side) $(N=9)$ & Group B (left side) $(N=8)$ & $P$ value \\
\hline \multicolumn{5}{|l|}{ Age (years) } \\
\hline Median \pm SDa (range) & $58 \pm 12.9(35-81)$ & $58 \pm 10.8(36-73)$ & $53.5 \pm 15.8(35-81)$ & 1.000 \\
\hline Gender & & & & 0.109 \\
\hline Male & $8(47.1 \%)$ & $6(66.7 \%)$ & $2(25 \%)$ & \\
\hline Female & $9(52.9 \%)$ & $3(33.3 \%)$ & $6(75 \%)$ & \\
\hline Histology & & & & 1.000 \\
\hline WD & $0(0 \%)$ & $0(0 \%)$ & $0(0 \%)$ & \\
\hline MD & $13(76.5 \%)$ & $7(77.8 \%)$ & $6(75 \%)$ & \\
\hline $\mathrm{PD}$ & $4(23.5 \%)$ & $2(22.2 \%)$ & $2(25 \%)$ & \\
\hline Site of metastasis & & & & 0.580 \\
\hline 1 & $6(35.3 \%)$ & $3(33.3 \%)$ & $3(37.5 \%)$ & \\
\hline 2 & $7(41.2 \%)$ & $3(33.3 \%)$ & $4(50.0 \%)$ & \\
\hline$\geq 3$ & $4(23.5 \%)$ & $3(33.3 \%)$ & $1(12.5 \%)$ & \\
\hline$B M I\left(k g / m^{2}\right)$ & & & & 0.871 \\
\hline Mean \pm SDa & $24.4 \pm 2.48$ & $23.4 \pm 2.98$ & $23.1 \pm 6.44$ & \\
\hline UGT1A1 & & & & 0.735 \\
\hline$* 1 / * 1$ & $15(88.2 \%)$ & $8(88.9 \%)$ & $7(87.5 \%)$ & \\
\hline$* 1 / * 28$ & $2(11.8 \%)$ & $1(11.1 \%)$ & $1(12.5 \%)$ & \\
\hline Response & & & & 0.067 \\
\hline Complete response & $0(0.0 \%)$ & $0(0 \%)$ & $0(0 \%)$ & \\
\hline Partial response & $2(11.8 \%)$ & $0(0 \%)$ & $2(25.0 \%)$ & \\
\hline Stable disease & $9(52.9 \%)$ & $7(77.8 \%)$ & $2(25.0 \%)$ & \\
\hline Progressive disease & $6(35.3 \%)$ & $2(22.2 \%)$ & $4(50.0 \%)$ & \\
\hline Responder & & & & 0.11 \\
\hline Yes & $2(11.8 \%)$ & $0(0 \%)$ & $2(25 \%)$ & \\
\hline No & $15(89.2 \%)$ & $9(100 \%)$ & $6(75 \%)$ & \\
\hline$D C R$ & & & & 0.23 \\
\hline Yes & $11(64.7 \%)$ & $7(77.8 \%)$ & $4(50 \%)$ & \\
\hline No & $6(35.3 \%)$ & $2(22.2 \%)$ & $4(50 \%)$ & \\
\hline
\end{tabular}

WD: well differentiated; MD: moderately differentiated; PD: poorly differentiated; DCR: disease control rate.

an increased likelihood of BRAF V600E mutation in Caucasian people [13, 22-25], our study provides different data in Asian patients with mCRC. Therefore, the absence of significant differences in age, sex, metastatic site, and tumor location may be attributable to the differences in the size or ethnicity of the sample population.
The standard first-line chemotherapeutic treatment for advanced $B R A F$-mutated $\mathrm{mCRC}$ is a fluoropyrimidinebased cytotoxic regimen, including either irinotecan or oxaliplatin combined with bevacizumab [26]. This combination was evaluated retrospectively in 127 patients with $B R A F$-mutated mCRC; the results revealed poor PFS of 6.4 
TABLE 2: Baseline characteristics of 17 patients with $B R A F$-mutated mCRC divided into irinotecan escalation and no irinotecan escalation groups.

\begin{tabular}{|c|c|c|c|}
\hline Characteristic & Group A (irinotecan escalation) $(N=8)$ & Group B (no irinotecan escalation) $(N=9)$ & $P$ value \\
\hline \multicolumn{4}{|l|}{ Age (years) } \\
\hline Median \pm SDa (range) & $59 \pm 11.9(36-72)$ & $51 \pm 14.6(35-81)$ & 0.459 \\
\hline Gender & & & 0.057 \\
\hline Male & $6(75 \%)$ & $2(22.2 \%)$ & \\
\hline Female & $2(25 \%)$ & $7(77.8 \%)$ & \\
\hline Histology & & & 0.576 \\
\hline WD & $0(0 \%)$ & $0(0 \%)$ & \\
\hline $\mathrm{MD}$ & $7(87.5 \%)$ & $6(66.7 \%)$ & \\
\hline $\mathrm{PD}$ & $1(12.5 \%)$ & $3(33.3 \%)$ & \\
\hline Site of metastasis & & & 0.959 \\
\hline 1 & $3(37.5 \%)$ & $3(33.3 \%)$ & \\
\hline 2 & $3(37.5 \%)$ & $4(44.5 \%)$ & \\
\hline$\geq 3$ & $2(25 \%)$ & $2(22.2 \%)$ & \\
\hline$B M I\left(k g / m^{2}\right)$ & & & 0.623 \\
\hline Mean $\pm \mathrm{SDa}$ & $23.9 \pm 3.47$ & $22.7 \pm 5.83$ & \\
\hline Response & & & 0.697 \\
\hline Complete response & $0(0 \%)$ & $0(0 \%)$ & \\
\hline Partial response & $1(12.5 \%)$ & $1(11.1 \%)$ & \\
\hline Stable disease & $5(62.5 \%)$ & $4(44.4 \%)$ & \\
\hline Progressive disease & $2(25 \%)$ & $4(44.4 \%)$ & \\
\hline Responder & & & 1.00 \\
\hline Yes & $1(12.5 \%)$ & $1(11.1 \%)$ & \\
\hline No & $7(87.5 \%)$ & $8(88.9 \%)$ & \\
\hline$D C R$ & & & 0.62 \\
\hline Yes & $6(75 \%)$ & $5(55.6 \%)$ & \\
\hline No & $2(25 \%)$ & $4(44.4 \%)$ & \\
\hline
\end{tabular}

TABLE 3: Toxicities of 17 patients with mCRC.

\begin{tabular}{lccc}
\hline Adverse effect & $\begin{array}{c}\text { Grades 1-2 } \\
(\%)\end{array}$ & $\begin{array}{c}\text { Grade } \geq 3 \\
(\%)\end{array}$ & $\begin{array}{c}\text { Any grade } \\
(\%)\end{array}$ \\
\hline Anemia & $13(76.5)$ & $0(0)$ & $13(76.5)$ \\
Fatigue & $10(58.8)$ & $0(0)$ & $10(58.8)$ \\
Nausea & $8(47.1)$ & $1(5.9)$ & $9(52.9)$ \\
Neutropenia & $8(47.1)$ & $1(5.9)$ & $9(52.9)$ \\
Hair loss & $8(47.1)$ & $0(0)$ & $8(47.1)$ \\
Thrombocytopenia & $6(35.3)$ & $0(0)$ & $6(35.3)$ \\
Abnormal liver & $6(35.3)$ & $0(0)$ & $6(35.3)$ \\
function & $3(17.6)$ & $1(5.9)$ & $4(23.5)$ \\
Vomiting & $3(17.6)$ & $0(0)$ & $3(17.6)$ \\
Acute kidney injury & $2(11.8)$ & $0(0)$ & $2(11.8)$ \\
Diarrhea & $2(11.8)$ & $0(0)$ & $2(11.8)$ \\
Paresthesia & & &
\end{tabular}

and 5.4 months with oxaliplatin- and irinotecan-based regimens plus bevacizumab as the first-line chemotherapy treatment, respectively $[10,27]$. To counter the disappointing result, the TRIBE study applied a more aggressive strategy of FOLFOXIRI (5-FU, leucovorin, oxaliplatin, and irinotecan) plus bevacizumab in a small subgroup of patients with $B R A F$-mutated CRC $[15,28]$ and revealed the median OS and PFS to be 19.0 and 7.5 months, respectively, compared with the OS of 10.7 and 5.5 months in the FOLFIRI plus bevacizumab group, respectively, after a median follow-up of 48.1 months (HR: 0.54, 95\% CI: 0.24-1.20 and HR: 0.57; 95\% CI: 0.27-1.23, respectively). Furthermore, the OS and PFS were more favorable in patients with right-sided mCRC on FOLFOXIRI plus bevacizumab than in those with left-sided mCRC on FOLFIRI plus bevacizumab, although the differences were not significant. Hence, the study concluded that FOLFOXIRI plus bevacizumab may be preferred as the first-line treatment for clinically selected patients with right-sided mCRC irrespective of their RAS and BRAF mutation status $[15,28]$. In our study, the median OS and PFS were 15.7 and 9.4 months, respectively, indicating favorable outcomes in patients with $B R A F$-mutated mCRC treated with irinotecan escalation strategy. Regarding tumor sidedness, although the differences were not significant, favorable PFS and OS trends were observed in patients with right-sided $\mathrm{mCRC}$, similar to the TRIBE study [15].

The improvement in applying the FOLFOXIRI regimen also increases toxicity. A phase III trial randomly assigned 244 patients to receive either infusional FOLFOXIRI or infusional FOLFIRI as the first-line treatment for mCRC. The FOLFOXIRI group experienced higher grade $2-3$ peripheral neurotoxicity ( $0 \%$ vs. $19 \% ; P<0.001$ ) and grade $3-4$ neutropenia $(28 \%$ vs. $50 \% ; P<0.001)$ than did the FOLFIRI group [29]. In the TRIBE study, the incidence of grade 3 or 4 neutropenia, diarrhea, stomatitis, and neurotoxicity (i.e., peripheral neuropathy) was significantly higher in the FOLFOXIRI plus bevacizumab group than in the FOLFIRI plus bevacizumab group [30]. In the present study, only 2 of $17(11.8 \%)$ patients experienced grade 3 toxicities from irinotecan escalation according to UGT1A1 genotyping. Because of tolerable 


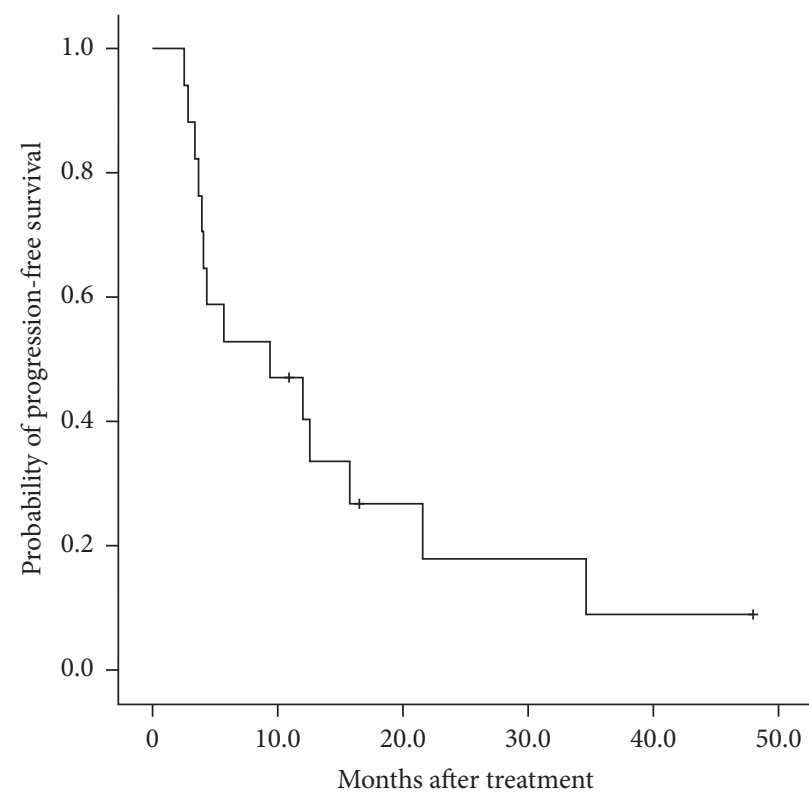

(a)

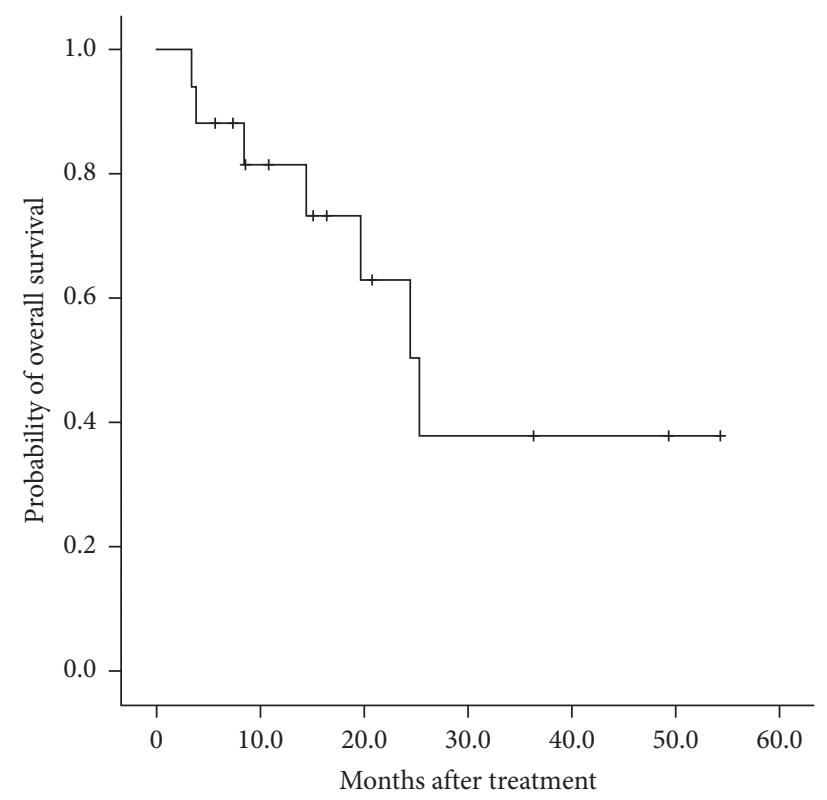

(b)

Figure 2: Kaplan-Meier survival curves for (a) median progress-free survival of 9.4 months and (b) median overall survival of 15.7 months for all 17 patients.

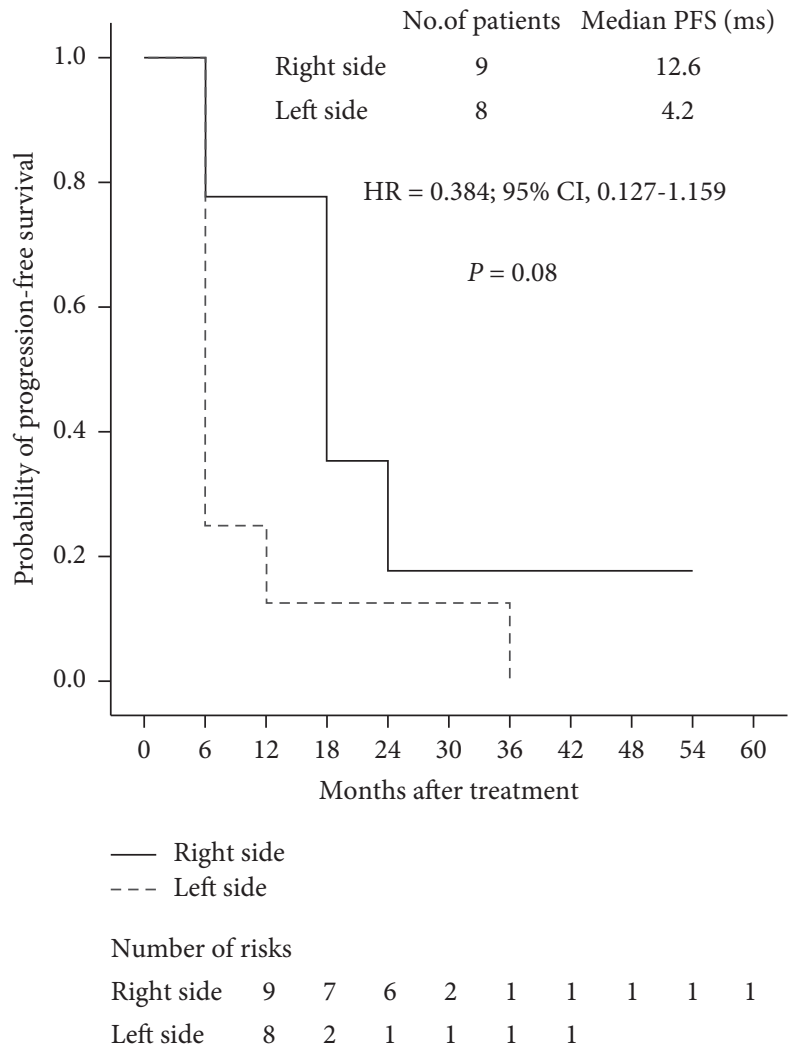

(a)

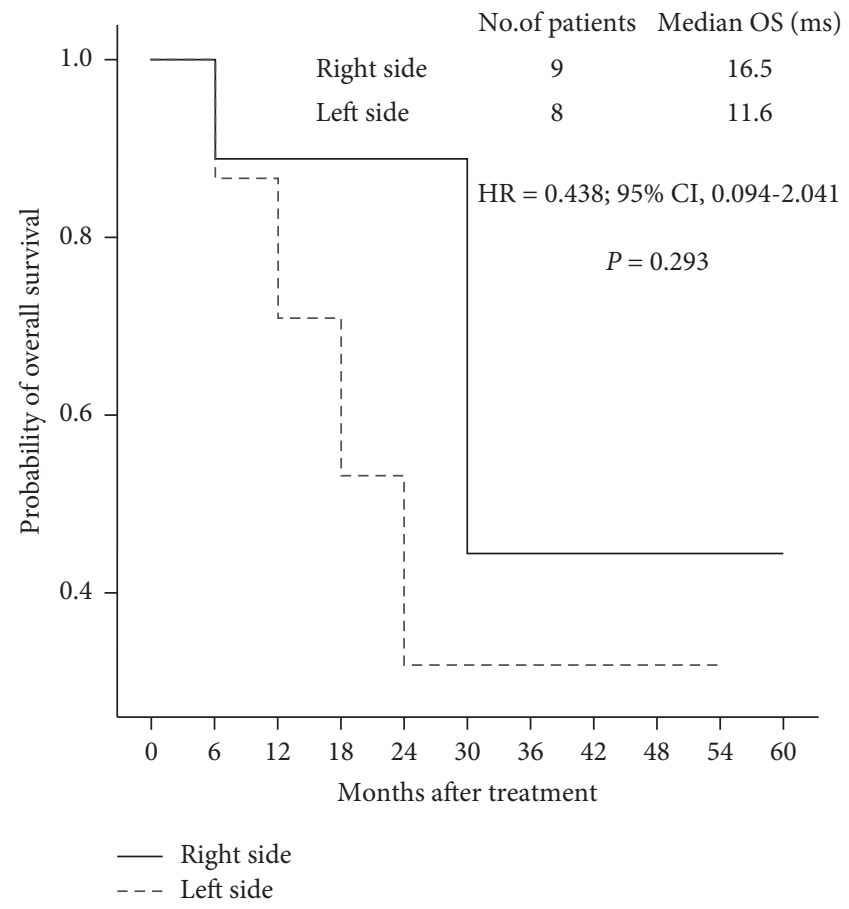

Number of risks

$\begin{array}{lllllllllll}\text { Right side } & 9 & 8 & 6 & 4 & 4 & 2 & 2 & 1 & 1 & 1\end{array}$ $\begin{array}{llllllllll}\text { Left side } & 8 & 6 & 4 & 3 & 1 & 1 & 1 & 1 & 1\end{array}$

(b)

Figure 3: Kaplan-Meier survival curves for (a) progress-free survival and (b) overall survival stratified by tumor sidedness. A trend of favorable PFS and OS was observed in patients with right-sided mCRC. 


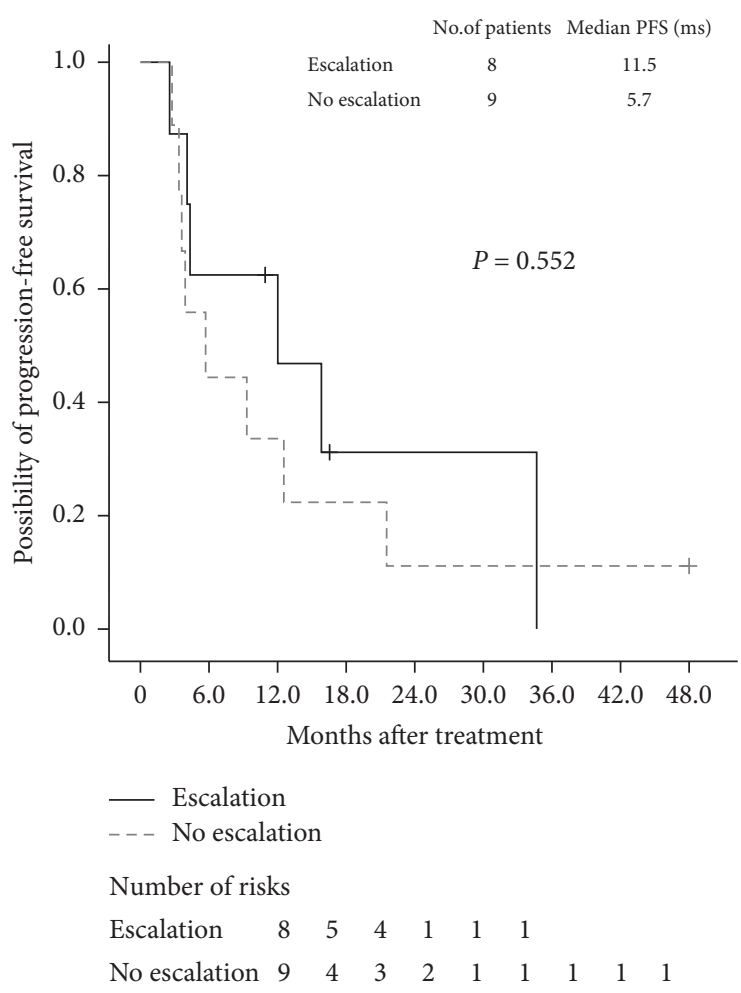

(a)

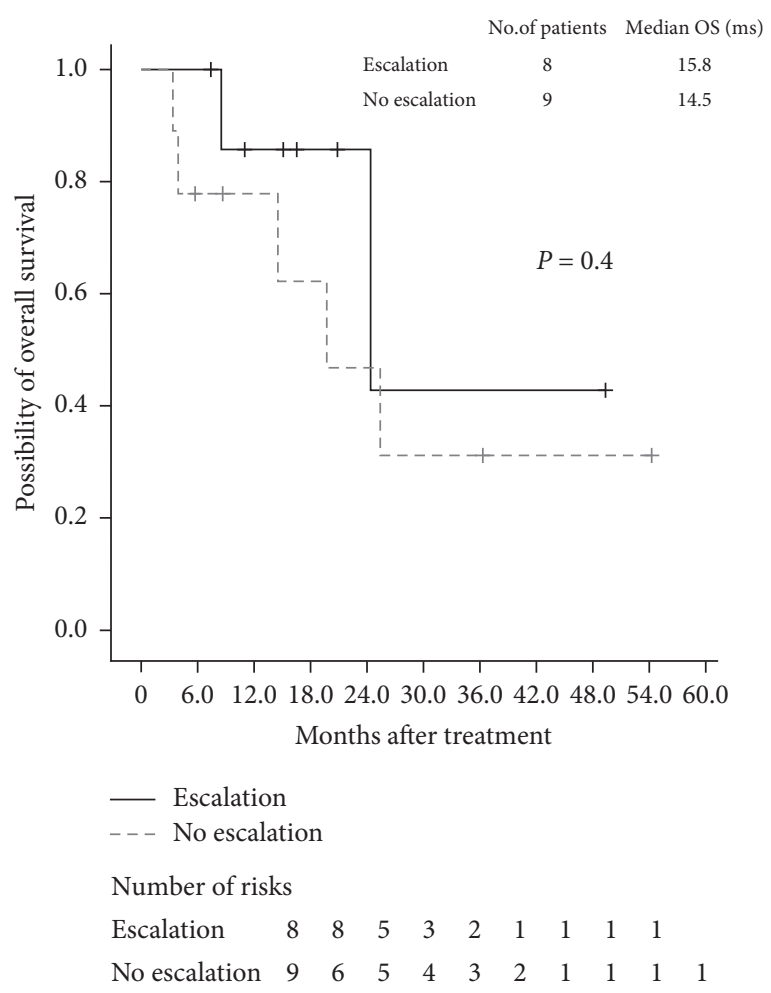

(b)

FIGURE 4: Kaplan-Meier survival curves for (a) progress-free survival and (b) overall survival stratified by irinotecan escalation or no escalation.

AEs, patients can proceed to second-line therapy with acceptable performance status after our first-line therapy. Irinotecan must be converted by a carboxylesterase to SN38 , which is actively cytotoxic and detoxified by the glucuronidation activity of UGT; therefore, the UGT1A1 genotype represents the development of drug-associated AEs. In a genotype-directed dose-determination study on irinotecan dose escalation in first-line FOLFIRI for mCRC, Marcuello et al. reported that compared with patients of other genotypes, patients with homozygous $U G T 1 A 1 * 28 / * 28$ developed more severe irinotecan-associated AEs more frequently [31]. By contrast, clinical presentations in patients with $U G T 1 A 1 * 1 / * 28$ exhibit individual variation; however, these patients generally tolerate the recommended initial irinotecan dose of $180 \mathrm{mg} / \mathrm{m}^{2}$ [32]. Conversely, patients with homozygous $U G T 1 A 1 * 1 / * 1$ genotype are more tolerant of irinotecan-associated AEs and can tolerate an irinotecan dose as high as $260 \mathrm{mg} / \mathrm{m}^{2}$ [32]. Patients with the $U G T 1 A 1 * 1 / * 1$ and $* 1 / * 28$ genotypes received high doses of irinotecan to achieve a favorable ORR without significant AEs in a phase II trial by Paez et al. [33]. According to the pan-Asian adapted ESMO consensus guidelines, patients with a favorable UGT1A1 genotype (homozygous wild $* 1 / * 1$ and heterozygous $* 1 / * 28$ ) can tolerate high-dose irinotecan without significant toxicity [34]. Here, no significant differences in PFS and OS were observed in patients in the escalation group, but a trend of better PFS and OS was found in our study. Future studies should include a larger sample size to verify the effect of irinotecan dose escalation.

Patients with $\mathrm{mCRC}$ who have pretherapeutic UGT1A1 genotyping and subsequent irinotecan dose adjustments tend to exhibit favorable responses and outcomes without a significant increase in toxicities when treated with FOLFIRI plus bevacizumab $[3,16,17,19]$. Compared with the TRIBE study results, our data reveal acceptable OS and PFS but less severe AEs. Moreover, $82.3 \%$ of the patients with BRAFmutated mCRC undergoing first-line treatment with this strategy could maintain this therapy with stable disease or proceed to second-line therapy in cases of disease progression, which means they still can preserve an acceptable performance status.

A second-line therapy using a combination of a $B R A F$ inhibitor or $M E K$ inhibitor with anti-EGFR may become the new standard of care for patients previously treated for BRAF V600E mutated CRC in cases of BRAF-mutated mCRC with aggressive characteristics [35]. The administration of triplet therapy led to approximately $50 \%$ grade 3 or 4 AEs; therefore, maintaining patients' performance status and encouraging them to undergo further therapy to prolong their lives is crucial [35].

The limitations of this study include its small sample size and data from a single center. Further prospective multicenter studies should be conducted to verify our results. 


\section{Conclusion}

In summary, the oncological outcomes of patients with $B R A F$-mutated mCRC treated using FOLFIRI plus bevacizumab with irinotecan dose escalation as a first-line therapy are acceptable with tolerable AEs; thus, it can be a feasible treatment option in selected patients.

\section{Data Availability}

The data used to support the findings of this study have been deposited in the DOI repository.

\section{Conflicts of Interest}

The authors declare that the research was conducted in the absence of any commercial or financial relationships that could be construed as potential conflicts of interest.

\section{Acknowledgments}

This work was supported by grants from the Ministry of Science and Technology (MOST 109-2314-B-037-035, MOST 109-2314B-037-040, and MOST 109-2314-B-037-046-MY3) and the Ministry of Health and Welfare (MOHW107-TDU-B-212123006, MOHW107-TDU-B-212-114026B, MOHW108-TDUB-212-133006, MOHW109-TDU-B-212-134026, and MOHW109-TDU-B-212-114006). The study was also funded by the Health and Welfare Surcharge of Tobacco Products, the Kaohsiung Medical University Hospital (KMUH109-9R32, KMUH109-9R33, KMUH109-9R34, KMUH109-9M30, KMUH109-9M31, KMUH109-9M32, KMUH109-9M33, KMUH-DK109003, KMUH-DK109005 3, KMUHS10903, and KMUHSA10903), Kaohsiung Medical University Center for Cancer Research (KMU-TC108A04-2), and a Kaohsiung Medical University Center for Liquid Biopsy and Cohort Research Center Grant (KMU-TC109B05). In addition, this study was supported by the Grant of Taiwan Precision Medicine Initiative, Academia Sinica, Taiwan. This manuscript was edited by Wallace Academic Editing.

\section{References}

[1] D. Barras, "BRAFV600E mutant colorectal cancer subtypes based on gene expression," Clinical Cancer Research, vol. 23, no. 1, pp. 104-115, 2017.

[2] S. Missiaglia, "Impact of BRAF and RAS mutations on firstline efficacy of FOLFIRI plus cetuximab versus FOLFIRI plus bevacizumab: analysis of the FIRE-3 (AIO KRK-0306) study," European Journal of Cancer, vol. 79, pp. 50-60, 2017.

[3] Y. S. Miller-Phillips et al., "Prospective analysis of UGT1A1 promoter polymorphism for irinotecan dose escalation in metastatic colorectal cancer patients treated with bevacizumab plus FOLFIRI as the first-line setting: study protocol for a randomized controlled trial," Trials, vol. 17, p. 46, 2016.

[4] A. P. Venook et al., "CALGB/SWOG 80405: phase III trial of irinotecan/5-FU/leucovorin (FOLFIRI) or oxaliplatin/5-FU/ leucovorin (mFOLFOX6) with bevacizumab (BV) or cetuximab (CET) for patients (pts) with KRAS wild-type (wt) untreated metastatic adenocarcinoma of the colon or rectum (MCRC)," Journal of Clinical Oncology, vol. 32, no. 15, 2014.
[5] A. Grassadonia, "Impact of primary tumor location in patients with RAS wild-type metastatic colon cancer treated with first-line chemotherapy plus anti-EGFR or anti-VEGF monoclonal antibodies: a retrospective multicenter study," Journal of Cancer, vol. 10, no. 24, pp. 5926-5934, 2019.

[6] H. Di Marino, "Treatment and survival outcome of BRAF -mutated metastatic colorectal cancer: a retrospective matched case-control study," Clinical Colorectal Cancer, vol. 17, no. 1, pp. e69-e76, 2018.

[7] E. Goode, "Prognostic implications of mismatch repair deficiency in patients with nonmetastatic colorectal and endometrial cancer," ESMO Open, vol. 4, no. 2, Article ID e000474, 2019.

[8] F. Petrelli, "Microsatellite instability and survival in stage II colorectal cancer: a systematic review and meta-analysis," Anticancer Research, vol. 39, no. 12, pp. 6431-6441, 2019.

[9] J. H. Ghidini, C. Wu, and T. Bekaii-Saab, "Targeting BRAF in metastatic colorectal cancer: maximizing molecular approaches," Cancer Treatment Reviews, vol. 60, pp. 109-119, 2017.

[10] J. Taieb, P. Laurent Puig, and A. Zaanan, "Exploring the best treatment options for BRAF-mutant metastatic colon cancer," British Journal of Cancer, vol. 121, no. 6, pp. 434-442, 2019.

[11] S. Lapeyre-Prost, A. Young, and F. McCormick, "Therapeutic strategies for targeting ras proteins," Genes \& Cancer, vol. 2, no. 3, pp. 359-372, 2011.

[12] C. N. Salt and E. S. Kopetz, "BRAF mutant colorectal cancer as a distinct subset of colorectal cancer: clinical characteristics, clinical behavior, and response to targeted therapies," Journal of Gastrointestinal Oncology, vol. 6, no. 6, pp. 660-667, 2015.

[13] B. Tran, "Impact of BRAF mutation and microsatellite instability on the pattern of metastatic spread and prognosis in metastatic colorectal cancer," Cancer, vol. 117, no. 20, pp. 4623-4632, 2011.

[14] F. Kopetz et al., "BRAF-mutated colorectal cancer: clinical and molecular insights," International Journal of Molecular Sciences, vol. 20, no. 21, 2019.

[15] C. Cremolini, "FOLFOXIRI plus bevacizumab versus FOLFIRI plus bevacizumab as first-line treatment of patients with metastatic colorectal cancer: updated overall survival and molecular subgroup analyses of the open-label, phase 3 TRIBE study," The Lancet Oncology, vol. 16, no. 13, pp. 1306-1315, 2015.

[16] C.-Y. Loupakis, "Prognostic advantage of irinotecan dose escalation according to uridine diphosphate glucuronosyltransferase 1A1 (UGT1A1) genotyping in patients with metastatic colorectal cancer treated with bevacizumab combined with 5-fluorouracil/leucovorin with irinotecan in a firstline setting," Translational Research, vol. 164, no. 2, pp. 169-176, 2014.

[17] C.-Y. Huang, "Clinical implication of UGT1A1 promoter polymorphism for irinotecan dose escalation in metastatic colorectal cancer patients treated with bevacizumab combined with FOLFIRI in the first-line setting," Translational Oncology, vol. 8, no. 6, pp. 474-479, 2015.

[18] L. L. Huang et al., "Characteristics and prevalence of KRAS, BRAF, and PIK3CA mutations in colorectal cancer by highresolution melting analysis in Taiwanese population," Clinica Chimica Acta, vol. 413, no. 19-20, pp. 1605-1611, 2012.

[19] H.-L. Tsai, C.-W. Huang, Y.-W. Lin et al., "Determination of the UGT1A1 polymorphism as guidance for irinotecan dose escalation in metastatic colorectal cancer treated with firstline bevacizumab and FOLFIRI (PURE FIST)," European Journal of Cancer, vol. 138, pp. 19-29, 2020. 
[20] P. Huang, S. G. Arbuck, E. A. Eisenhauer et al., "New guidelines to evaluate the response to treatment in Solid tumors," JNCI: Journal of the National Cancer Institute, vol. 92, no. 3, pp. 205-216, 2000.

[21] M. Ducreux, A. Chamseddine, P. Laurent-Puig et al., "Molecular targeted therapy of BRAF-mutant colorectal cancer," Therapeutic Advances in Medical Oncology, vol. 11, Article ID 1758835919856494, 2019.

[22] F. Loupakis, R. Moretto, G. Aprile et al., "Clinico-pathological nomogram for predicting BRAF mutational status of metastatic colorectal cancer," British Journal of Cancer, vol. 114, no. 1, pp. 30-36, 2016.

[23] T. Jeanne, G. Peter, L. Lara et al., "Optimizing targeted therapeutic development: analysis of a colorectal cancer patient population with the BRAFV600E mutation," International Journal of Cancer, vol. 128, no. 9, pp. 2075-2084, 2011.

[24] C. Gibbs, C. E. Atreya, and K. Van Loon, "Emerging treatment options for BRAF-mutant colorectal cancer," Gastrointestinal Cancer: Targets and Therapy, vol. 8, pp. 13-23, 2018.

[25] H. H. Yoon, "Racial differences in BRAF/KRAS mutation rates and survival in stage III colon cancer patients," Journal of the National Cancer Institute, vol. 107, no. 10, 2015.

[26] R. Cohen, "BRAF-mutated colorectal cancer: what is the optimal strategy for treatment?" Curr Treat Options Oncol, vol. 18, no. 2, p. 9, 2017.

[27] V. K. Morris, M. J. Overman, J. Zhi-Qin et al., "Progressionfree survival remains poor over sequential lines of systemic therapy in patients with BRAF-mutated colorectal cancer," Clinical Colorectal Cancer, vol. 13, no. 3, pp. 164-171, 2014.

[28] C. Cremolini, C. Antoniotti, S. Lonardi et al., "Primary tumor sidedness and benefit from FOLFOXIRI plus bevacizumab as initial therapy for metastatic colorectal cancer. Retrospective analysis of the TRIBE trial by GONO," Annals of Oncology, vol. 29, no. 7, pp. 1528-1534, 2018.

[29] A. Antoniotti, R. Sergio, I. Brunetti et al., "Phase III trial of infusional fluorouracil, leucovorin, oxaliplatin, and irinotecan (FOLFOXIRI) compared with infusional fluorouracil, leucovorin, and irinotecan (FOLFIRI) as first-line treatment for metastatic colorectal cancer: the Gruppo Oncologico Nord Ovest," Journal of Clinical Oncology, vol. 25, no. 13, pp. 1670-1676, 2007.

[30] L. Fotios, C. Chiara, M. Gianluca et al., "Initial therapy with FOLFOXIRI and bevacizumab for metastatic colorectal cancer," New England Journal of Medicine, vol. 371, no. 17, pp. 1609-1618, 2014

[31] E. Marcuello, D. Páez, L. Paré et al., "A genotype-directed phase I-IV dose-finding study of irinotecan in combination with fluorouracil/leucovorin as first-line treatment in advanced colorectal cancer," British Journal of Cancer, vol. 105, no. 1, pp. 53-57, 2011.

[32] C. S. Páez, J. Marshall, and J. Barrueco, "Randomized, controlled trial of irinotecan plus infusional, bolus, or oral fluoropyrimidines in first-line treatment of metastatic colorectal cancer: updated results from the BICC-C study," Journal of Clinical Oncology, vol. 26, no. 4, pp. 689-690, 2008.

[33] D. Páez, M. Tobena, J. Fernández-Plana et al., "Pharmacogenetic clinical randomised phase II trial to evaluate the efficacy and safety of FOLFIRI with high-dose irinotecan (HDFOLFIRI) in metastatic colorectal cancer patients according to their UGT1A 1 genotype," British Journal of Cancer, vol. 120, no. 2, pp. 190-195, 2019.

[34] T. Yoshino, D. Arnold, H. Taniguchi et al., "Pan-Asian adapted ESMO consensus guidelines for the management of patients with metastatic colorectal cancer: a JSMO-ESMO initiative endorsed by CSCO, KACO, MOS, SSO and TOS," Annals of Oncology, vol. 29, no. 1, pp. 44-70, 2018.

[35] E. Arnold, "Binimetinib, encorafenib, and cetuximab triplet therapy for patients with BRAF V600e-mutant metastatic colorectal cancer: safety lead-in results from the phase III BEACON colorectal cancer study," Journal of Clinical Oncology, vol. 37, no. 17, pp. 1460-1469, 2019. 